\title{
Dedo pulgar en gatillo bilateral, tratamiento quirúrgico y conservador en paciente de 4 años de edad.
}

Bilateral trigger finger, surgical and conservative treatment in a 4 year old patient.

Polegar em gatilho bilateral, tratamento cirúrgico e conservador em paciente de quatro anos de idade.

Guillermo Andrés Bretón Gómez, MD. ${ }^{1}$ (D), John Jairo Vargas Rueda, MD. ${ }^{2}$ (D), Paola Andrea Ardila Forero, MD. ${ }^{3}$ (D), Andrea López Villegas, $\mathrm{MD}^{4}$ (D)

1. Médico General, IPS Centro Clínico Carvajal, Sabana de Torres, Santander, Colombia.

2. Médico General, Residente cuarto año Cirugía Plástica y Reconstructiva, Universidad Militar Nueva Granada, Bogotá, Colombia.

3. Médica General, Servicio de Urgencias Hospital Central de la Policía Nacional, Bogotá, Colombia.

4. Médica General, SSO Escuela de Aviación Policía Nacional. Bogotá, Colombia.

Correspondencia. Guillermo Andrés Bretón Gómez. Médico General, IPS Centro Clínico Carvajal, Sabana de Torres, Santander, Colombia. Tel.3208008119.Email.guillermobreton93@gmail.com

Cómo citar: Bretón Gómez GA, Vargas Rueda JJ, Ardila Forero PA, López Villegas A. Dedo pulgar en gatillo bilateral, tratamiento quirúrgico y conservador en paciente de 4 años de edad. MedUNAB. 2020;23(1):131-136. doi:10.29375/01237047.3616.

\section{INFORMACIÓN DEL ARTÍCULO:}

Artículo recibido: 17 de mayo de 2019

Artículo aceptado: 09 de enero de 2020

Doi: https://doi.org/10.29375/01237047.3616

\section{RESUMEN}

Introducción. El dedo en gatillo es una anomalía infrecuente en niños que afecta principalmente al dedo pulgar, de etiología desconocida. El manejo puede ser conservador o quirúrgico. Reporte de caso. Paciente pediátrica escolar de 4 años y 7 meses de edad es remitida al servicio de cirugía plástica por deformidad en flexión fija del pulgar derecho, de 4 meses de evolución asociada a nódulo palpable. Se interviene quirúrgicamente de forma efectiva, sin complicaciones, secuelas ni recurrencia. Discusión. La paciente fue manejada quirúrgicamente de forma efectiva. 
Se hizo un seguimiento durante 3 años con una evolución satisfactoria, teniendo una recuperación total de la función del pulgar, sin secuelas y adecuada adaptabilidad al medio. Conclusión. Este artículo tiene como objetivo presentar un caso clínico que busca llamar la atención sobre las indicaciones del tratamiento conservador versus quirúrgico que existen en la literatura, corresponde a una paciente pediátrica de nuestro medio con dedo pulgar en gatillo bilateral, su manejo y los resultados postoperatorios. El dedo pulgar en gatillo pediátrico tiene una baja prevalencia en el mundo. No hay estudios de incidencia ni prevalencia en nuestro medio. La edad de presentación es variable, los signos y síntomas no son iguales a los del adulto, el compromiso puede ser bilateral, su diagnóstico es clínico y el tratamiento quirúrgico, dependiendo del grado de compromiso, puede ser el más efectivo.

Palabras claves:

Trastorno del Dedo en Gatillo; Anomalías Congénitas; Lactante; Recién Nacido; Tratamiento Conservador; Terapéutica; Complicaciones Posoperatorias.

\section{ABSTRACT}

Introduction. Trigger finger is a rare anomaly in children; it is of unknown etiology and mainly affects the thumb. Management may be conservative or surgical. Case report. Pediatric patient of 4 years and 7 months of age is referred to plastic surgery service for fixed flexion deformity of the right thumb, after 4 months of evolution associated with a palpable nodule. It is successfully intervened surgically, without complications, sequels or recurrence. Discussion. The patient was effectively treated via surgery. Follow-up was carried out for three years with satisfactory evolution, showing full recovery of the function of the thumb, with no after-effects and with adequate adaptability to the environment. Conclusion. The objective of this article is to present a clinical case that aims to draw attention to the indications for conservative versus surgical treatment existing in the literature, corresponding to a pediatric patient from our milieu with a bilateral trigger finger, its treatment and postoperative results. Pediatric trigger finger has low prevalence in the world. There are no incidence or prevalence studies in the cases that were reviewed. The age at which it arises is variable; the signs and symptoms are not the same as those of adults; involvement may be bilateral; diagnosis is clinical and surgery may be the most effective treatment, depending on the degree of involvement.

Keywords:

Trigger Finger Disorder; Congenital Abnormalities; Newborn Infant; Conservative Treatment; Therapeutics; Postoperative Complications.

\section{RESUMO}

Introdução. $\mathrm{O}$ dedo em gatilho é uma anomalia incomum em crianças, que afeta principalmente o polegar, de etiologia desconhecida. O tratamento pode ser conservador ou cirúrgico. Relato de caso. Paciente pediátrica de quatro anos e sete meses de idade é encaminhada ao serviço de cirurgia plástica para deformidade em flexão fixa do polegar direito, com quatro meses de evolução associada a nódulo palpável. Foi tratada cirurgicamente de forma eficaz, sem complicações, sequelas ou recorrência. Discussão. A paciente foi tratada cirurgicamente de forma eficaz. Foi feito um acompanhamento por três anos com uma evolução satisfatória, com recuperação total da função do polegar, sem sequelas e adaptabilidade adequada ao ambiente. Conclusão. Este artigo tem como objetivo apresentar um caso clínico que busca chamar a atenção para as indicações do tratamento conservador versus cirúrgico, existentes na literatura, que corresponde a uma paciente pediátrica em nosso meio com um polegar de gatilho bilateral, seu manejo e resultados pós-operatórios. O polegar em gatilho pediátrico tem uma baixa prevalência no mundo. Não há estudos de incidência ou prevalência nos casos consultados. A idade de apresentação é variável, os sinais e sintomas não são iguais aos do adulto, o comprometimento pode ser bilateral, o diagnóstico é clínico e o tratamento cirúrgico, dependendo do grau de comprometimento, pode ser o mais eficaz.

Palavras-chave:

Dedo em Gatilho; Anomalidades Congênitas; Lactente; Recém-Nascido; Tratamento Conservador; Terapêutica; Complicações Pós-Operatórias. 


\section{Introducción}

La tenosinovitis estenosante, o 'dedo en gatillo', fue descrita por primera vez en pacientes adultos en 1850 por el doctor Alphonse Notta, que observó la presencia de un nódulo en el tendón flexor que limitaba los movimientos normales, ubicado al mismo nivel de la unión Metacarpofalángica (unión MCF) (1). Patológicamente se caracteriza por un engrosamiento del flexor pollicis longus y la polea anular, ocasionado por una proliferación y engrosamiento sinovial, seguidos de la formación de un nódulo (Nódulo de Notta) a nivel de la unión MCF que impide el correcto deslizamiento de dicho tendón (2).

En niños, es una patología infrecuente, su incidencia varía de $0.05 \%$ a $0.3 \%$, con una prevalencia de 3.3 por cada 1,000 niños (3). Representa solo el $2.2 \%$ de todas las anomalías congénitas del miembro superior pediátrico. No obstante, es el procedimiento quirúrgico en manos infantiles que se realiza con más frecuencia. De forma unilateral, compromete al pulgar en más del $90 \%$ de los casos. Sin embargo, alrededor del $25 \%$ es de casos bilaterales, sin diferencias significativas en sexo ni lateralidad (4). La etiología exacta es aún desconocida. Varios autores han sostenido que es una condición congénita (5). Otros, en estudios más recientes, consideran que es adquirida, y la relacionan de forma potencial a traumatismo y succión digital (6). De forma menos frecuente es asociada a sesamoideos prominentes o secundario a variaciones anatómicas (4). El compromiso de los otros dedos de la mano es 10 veces menos común y se considera una entidad diferente (7).

Clínicamente, se evidencia una deformidad en flexión de la Articulación Interfalángica (IF) del pulgar y un nódulo palpable en la unión MCF, en el $82 \%$ y $96 \%$ de los casos, respectivamente. El tratamiento puede ser conservador, con el fin de obtener una resolución espontánea; o quirúrgico. Esta decisión dependerá de la edad de aparición y la severidad del cuadro clínico (8).

Este artículo tiene como objetivo presentar un caso clínico y una breve revisión de la literatura que busca llamar la atención sobre las indicaciones del tratamiento conservador versus el quirúrgico, ya que, a pesar de ser una entidad infrecuente en el mundo, es uno de los principales motivos de consulta para el cirujano de manos, siendo el tratamiento quirúrgico de esta afección el procedimiento más frecuente realizado en la mano infantil.

\section{Presentación de caso}

Paciente pediátrica escolar de 4 años y 7 meses de edad con antecedente familiar de hermana mayor con diagnóstico de dedo pulgar de la mano derecha en gatillo a los 7 meses de edad, que fue resuelto quirúrgicamente al completar el año de edad. La paciente fue remitida al servicio de cirugía plástica por presentar deformidad en flexión permanente del pulgar de la mano derecha y engatillamiento ocasional del pulgar de la otra mano. Los padres notaron la deformidad bilateral alrededor de los 4 años de edad. En el pulgar derecho, el grado de deformidad en flexión era mayor. La madre extendía pasivamente ambos pulgares, pero el derecho retornaba rápidamente a la flexión, mientras que el izquierdo lo hacía de forma ocasional. En el examen físico se encontró flexión fija de $30^{\circ}$ e incapacidad para la extensión, tanto pasiva como activa de la IF del primer dedo de la mano derecha, asociado a la presencia de un nódulo palpable a nivel de la unión MCF del mismo dedo, de aproximadamente $1.5 \mathrm{~cm}$ x $1.5 \mathrm{~cm}$ (figura 1); el primer dedo de la mano izquierda mostraba un engatillamiento ocasional, sin más hallazgos.

El diagnóstico fue 'dedo en gatillo bilateral'. Se decidió intervenir quirúrgicamente el dedo pulgar derecho y dar manejo conservador al dedo contralateral con ciclo corto suministrando antiinflamatorios. Se programó cirugía electiva y ambulatoria según disponibilidad de salas. Al mes siguiente la paciente es citada con paraclínicos prequirúrgicos para la intervención.

Figura 1. A. Deformidad fija en flexión.

B. Imposibilidad para la extensión pasiva

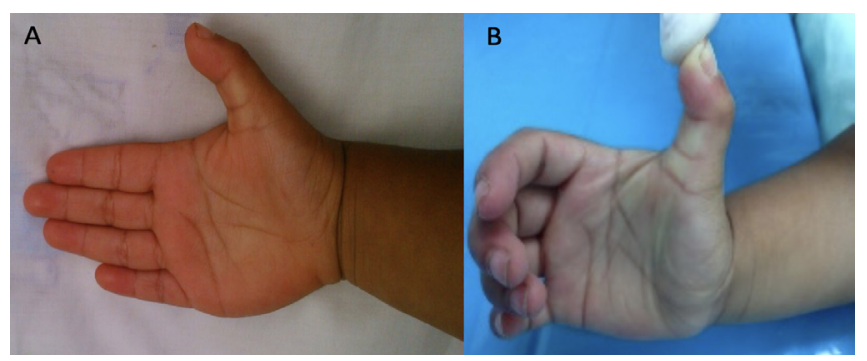

Fuente. Elaboración de los autores

\section{Hallazgos:}

1. Nódulo en la base del dedo pulgar de la mano derecha.

2. Incapacidad para la flexión completa de la unión MCF del primer dedo.

3. Bajo anestesia general no se alcanza la excursión completa del tendón flexor en pasivo y activo.

Previa asepsia y antisepsia, bajo anestesia general, en decúbito supino y profilaxis antibiótica, se realiza la exanguinación del miembro superior derecho. Se ubica un torniquete manual y se identifican los hallazgos descritos. Posteriormente, con bisturí, se realiza una incisión transversa a nivel de la unión MCF en la cara volar del dedo pulgar de la mano derecha. Se identifica estenosis de la polea A1 por inflamación crónica, tanto de la polea como del Flexor Pollicis Longus FPL, siendo marcado el aumento del tamaño de este último, con un 
Nódulo de Notta identificado macroscópicamente (figura 2). Se realiza una disección por planos, se preservan los paquetes neurovasculares radial y cubital del dedo pulgar, se hace una incisión vertical en la polea A1 para hacer tenolísis del FPL obteniendo excursión completa en flexoextensión pasiva y activa de la unión MCF. Posteriormente se revisa hemostasia, cierre por planos, tejido celular subcutáneo con Vicryl $5.0 \mathrm{y}$ piel con sutura con 9 puntos simples separados de Monocryl (figura 2). Posteriormente se retira el torniquete, se revisa perfusión distal del dedo, el llenado capilar adecuado y se deja envuelto en gasas alcoholadas, gasas secas y vendaje bultoso.

Figura 2. A. Estenosis de la polea A1. B. Nódulo de Notta. C. Extensión pasiva completa

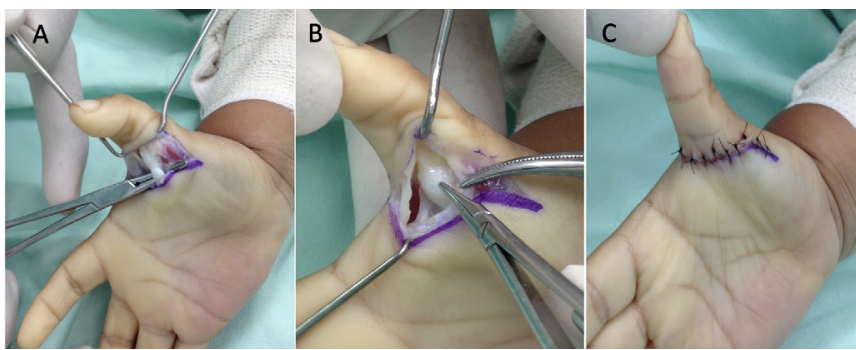

Fuente. Elaboración de los autores

La paciente es dada de alta con analgesia y profilaxis antibiótica. En la valoración postoperatoria al mes de la operación presenta evolución satisfactoria, así como en la siguiente valoración, 4 años más tarde (figura 3).

Figura 3. A. Cicatriz imperceptible. B. Pulgar en extensión. C. Manos en extensión completa.

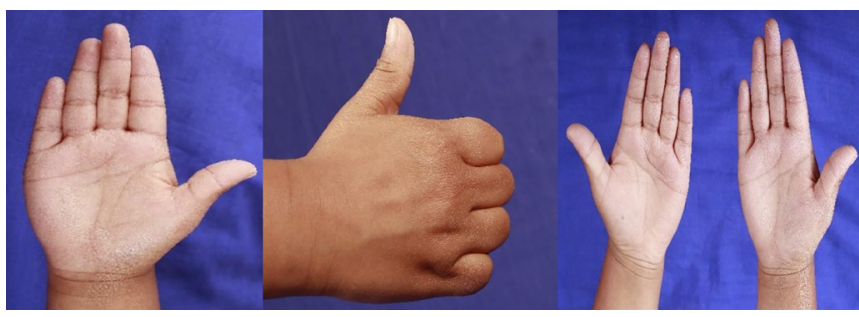

Fuente. Elaboración de los autores

\section{Discusión}

El dedo pulgar en gatillo es una patología relativamente infrecuente en la población pediátrica, de predominio unilateral, aunque el compromiso bilateral también es significativo: alrededor del $25 \%$ al $30 \%$ (8-10). Frecuentemente, los pacientes presentan una deformidad en flexión del pulgar que puede ser o no corregible y que está asociada a la presencia del Nódulo de Notta, el engatillamiento, dolor y chasquido son hallazgos frecuentes (8,10-11). En la paciente el compromiso fue bilateral con los signos más frecuentes reportados en la literatura y descritos previamente.

Rekha (3) reportó un caso de una niña de 6 años de edad con dedo pulgar en gatillo bilateral. Sus padres notaron la deformidad cuando tenía 6 meses de vida. Ellos podían extender los pulgares completamente, pero a los 5 años de edad, la flexión se hizo fija y al intentar extenderlos se tornó doloroso. Fue llevada cirugía y se encontró, además de la tenosinovitis en la polea A1, compromiso en la Av (3). Los signos clínicos encontrados son similares al caso clínico acá estudiado: ambos pulgares sintomáticos desde la presentación clínica inicial. Sin embargo, en el caso que acá se revisa, la paciente tuvo un inicio más tardío, con mayor compromiso del pulgar derecho a pesar del inicio sincrónico y nunca presentó dolor, siendo este un síntoma infrecuente.

La etiología permanece desconocida y controversial. Algunos estudios soportan la hipótesis congénita, debido a la presencia de esta alteración desde el nacimiento y también por reportes de casos en gemelos monocigotos, que presentaban un sugestivo patrón autosómico dominante. Se han realizado múltiples estudios prospectivos, con una muestra colectiva total de 14,581 recién nacidos, y no se ha encontrado ningún caso $(12,6)$. Algunos autores sugieren que la típica posición en flexión del pulgar en el periodo prenatal y neonatal provoca una degeneración del colágeno y proliferación sinovial, produciendo un nódulo del FPL y de la vaina que lo recubre. Buchman et al, en un estudio de microscopía electrónica analizando la polea A1 y el Nódulo de Notta en 9 pacientes con dedo pulgar en gatillo, observó gran cantidad de colágeno maduro y fibroblastos a nivel del FPL y la vaina, sin cambios inflamatorios o degenerativos, descartando potencialmente una causa infecciosa, inflamatoria o degenerativa (13). En un estudio más reciente realizado en 25 especímenes de poleas A1 por Khoshhal et al (4), se evidenció la presencia de citoproteinas contráctiles y miofibroblastos, lo cual sugiere una proliferación del tejido fibroso causante de la tenosinovitis, que puede ocurrir tanto en la fase intrauterina como extrauterina del desarrollo. Si el proceso inicia in útero, una constante posición flexionada del pulgar puede causar degeneración del colágeno y proliferación sinovial, causando que el paciente puede presentar una contractura en flexión fija y si ocurre de forma extrauterina, puede presentar engatillamiento y luego, con la maduración del tejido fibroso resulta en una flexión fija. En recién nacidos se puede retrasar o dificultar el diagnóstico debido a la actitud en flexión normal del pulgar durante los primeros tres meses de vida (14).

Huwae et al presentaron recientemente el caso de una paciente de 3 años de edad con dedo pulgar en gatillo bilateral presente desde el nacimiento (6), con deformidad en flexión fija en ambos pulgares no corregible de forma pasiva. El caso 
se presentaba sin antecedente familiar ni otros hallazgos, con un estadio 3, según la clasificación de Watanabe (15). Diagnosticada con dedo pulgar en gatillo bilateral congénito y corregido quirúrgicamente con la liberación bilateral de las poleas A1. Este reporte pone en consideración la posible ambigüedad de la etiología del dedo pulgar en gatillo, tanto la congénita inherente al caso previamente descrito, como una causa adquirida en el caso clínico acá estudiado: el pulgar derecho de la paciente se encontraba en estadio 3, mientras que el pulgar izquierdo en estadio 1 .

Aunque la causa adquirida de igual manera no se ha dilucidado, es la más frecuente reportada y corresponde con las características del actual caso clínico $(2,12,16)$. Existen diferentes opciones de tratamiento conservador, entre las que incluyen observación, terapia física y el uso de férulas. Baek et al. reportaron que el dedo pulgar en gatillo se puede resolver espontáneamente en más del $60 \%$ de los pacientes en un promedio de 48 meses. Posteriormente, Baek et al demostraron una resolución espontánea en más del 75\% de los casos (17). Forlin et al reportaron resultados satisfactorios en el $77 \%$ de los casos en un seguimiento mínimo de 5 años (18). En un estudio retrospectivo, 107 pulgares fueron observados por al menos 6 meses, y la resolución espontánea fue solo del 12\% (12). La eficacia del manejo conservador con terapia física no está bien establecida. Watanabe et al reportaron resultados del tratamiento con ejercicios de extensión pasiva en 48 niños con 60 pulgares en gatillo con diagnóstico promedio a los 2 años de edad. A pesar de que el $96 \%$ de los pulgares mostraron un resultado satisfactorio, el $59 \%$ persistieron con movimientos anormales (15). Nemoto et al trataron a 30 pacientes con 40 pulgares en gatillo con férula dorsal en extensión con un seguimiento de 10 meses. Los autores observaron resolución completa en el $60 \%$ de los casos. Tan et al reportaron una tasa de efectividad del tratamiento conservador con ejercicios de extensión pasiva en casa y terapia física combinada con férula del $66 \%$ la cual pierde efectividad a medida que aumenta la edad (del $89 \%$ en niños menores de 1 año al $50 \%$ en mayores de 3 años). La efectividad de la terapia con férula fue mayor que la extensión pasiva en casa, con un 52\% y un $77 \%$ respectivamente (19). A pesar de que se ha comprobado el beneficio de corticoides en adultos, y que algunos autores hayan encontrado buenos resultados con el uso de estos en niños menores de 3 años, recientemente no se ha usado ni recomendado alguno en niños (18).

En la literatura consultada, la efectividad del tratamiento conservador es variable: existen limitaciones de este debido a que la mayoría son estudios retrospectivos, con unas muestras pequeñas de casos con seguimientos cortos, pero es una opción de tratamiento potencialmente válida en algunos pacientes. El pulgar izquierdo de la paciente presentaba engatillamiento ocasional, no había deformidad en flexión fija, y se indicó manejo conservador con ejercicios de extensión pasiva en casa, que fue realizada por la madre y presentó una mejoría completa a los 2 meses, aproximadamente, sin deformidad residual.

Las indicaciones definitivas del tratamiento quirúrgico son el engatillamiento doloroso y deformidad rígida del pulgar (10). Otras indicaciones quirúrgicas tenidas en cuenta son presentadas por los niños mayores de un año que no mejoran con el tratamiento conservador. En niños mayores de 3 años y cuando la patología es bilateral (3), y consiste en la liberación por vía palmar de la polea A1 (20). El tratamiento quirúrgico ha mostrado ser el más efectivo (10). Tan et al revisaron 72 pulgares que fueron llevados a cirugía. La recurrencia de estos fue del $1.4 \%$ a los 7 meses probablemente por una inadecuada liberación de la polea $\mathrm{A} 1$, y con una tasa de infecciones del $2.8 \%$ que mejoraron con antibióticos orales (19). La edad en la cual se debe realizar el procedimiento quirúrgico no está establecida. Sin embargo, en los niños mayores de tres años de edad, aumenta el riesgo de flexión residual (14,21). El uso de liberación percutánea no se recomienda en niños, por el mayor riesgo de lesión neurovascular y de liberación incompleta $(22,23)$.

El pulgar derecho de la paciente tenía una evidente indicación quirúrgica dada principalmente por la deformidad fija del mismo. Usualmente la estenosis está confinada a la polea A1, pero ocasionalmente la polea anular Av localizada entre la A1 y la oblicua, puede estar presente. Este es el tipo de caso reportado por Rekha (3). En el caso del presente artículo, en cambio, la liberación de la polea A1 fue suficiente para lograr la extensión completa del pulgar derecho. En contraste con la relativamente baja efectividad del manejo conservador, en el pulgar izquierdo de la paciente fue útil y recuperó totalmente su funcionalidad.

\section{Conclusión}

El dedo pulgar en gatillo pediátrico es una alteración del aparato flexor, caracterizada por un engatillamiento $\mathrm{y}$ posterior bloqueo en flexión del pulgar que puede ser unilateral o bilateral. Es relativamente infrecuente en niños, con una baja prevalencia e incidencia en el mundo. La epidemiología en Colombia y Latinoamérica es desconocida. $\mathrm{Su}$ etiología permanece desconocida y controversial, la edad promedio de presentación es alrededor de los 2 años de edad, clínicamente caracterizada por la presencia de una deformidad en flexión del pulgar que puede ser fija o móvil, típicamente no dolorosa, asociada a la presencia del nódulo de Notta ubicado a nivel de la MCF. El diagnóstico es completamente clínico. El tratamiento puede ser conservador en ciertos pacientes o quirúrgico que hasta el momento es el tratamiento más efectivo. La paciente de este caso clínico se benefició totalmente de ambas conductas. 


\section{Consideraciones éticas}

Existe consentimiento informado firmado por los padres de la paciente para la publicación de este reporte de caso y de las imágenes.

\section{Conflicto de interés}

Los autores declaran que no existe conflicto de interés con respecto a la publicación de este artículo.

\section{Referencias}

1. Flatt A. Notta's nodules and trigger digits. Baylor University Medical Center Proceedings. 2007; 20(2):143-145. doi: https://doi.org/10.1080/08998280.2007.11928272

2. Kuo M, Rayan G. Complete annular and partial oblique pulley release for pediatric locked trigger thumb. Hand 5. 2010; 5(4):408-414. doi: https://doi.org/10.1007/s11552-010-9274$\underline{8}$

3. Rekha Y. Delayed Case of Congenital Bilateral Trigger Thumb: A Case Report and Review of Literature. J Orthop Case Rep. 2014; 4(1):24-27. doi: https://doi.org/10.13107/ jocr.2250-0685.143

4. Khoshhal K, Jarvis J, Uhthoff H. Congenital trigger thumb in children: electron microscopyand immunohistochemical analysis of the first annular pulley. Journal of Pediatric Orthopaedics B. 2012; 21(4):295-299. doi: https://doi. org/10.1097/BPB.0b013e3283536962

5. Kikuchi N, Ogino T. Incidence and Development of Trigger Thumb in Children. The Journal of Hand Surgery. 2006; 31(4):541-543. doi: https://doi.org/10.1016/j. 近sa.2005.12.024

6. Huwae T, Bastian J, Lukman R. Congenital bilateral trigger thumb in 3 years old girl: A case report. International Journal of Surgery Open. 2018; 13:15-19. doi: https://doi.org/10.1016/j. ijso.2018.07.004

7. Schaverien M, Godwin Y. Paediatric trigger finger: Literature review and management algorithm. Journal of Plastic, Reconstructive \& Aesthetic Surgery. 2011; 64(5):623-631. doi: https://doi.org/10.1016/j.bjps.2010.09.011

8. Leung O, Ip F, Wong T, Wan S. Trigger thumbs in children: results of surgical release. hongMedJ. 2011; 17(5):372-375. pmid: 21979473

9. Herdem M, Bayram H, Toðrul E, Sarpel Y. Clinical analysis of the trigger thumb of childhood. Turk J Pediatr 2003; 45(3):237239. pmid: 14696802

10. Twu J, Angeles J. Developmental Trigger Thumb. Pediatr Ann. 2016; 45(4):135-138. doi: https://doi.org/10.3928/0090448120160317-02

11. Wang E, Xu X, Dagum, A. Mirror-image Trigger Thumb in Dichorionic Identical Twins. Orthopedics. 2012; 35(6):981-
983. doi: https://doi.org/10.3928/01477447-20120525-48

12. Bae D, Shah A. Management of Pediatric Trigger Thumb and Trigger Finger. Journal of the American Academy Orthopaedic Surgeons. 2012;20(4):206-213 doi: https://doi.org/10.5435/ JAAOS-20-04-206

13. Buchman MT, Gibson TW, McCallum D, Cuda DD, Ramos AG. Transmission electron microscopic pathoanatomy of congenital trigger thumb. Journal of Pediatric Orthopaedics. 1999; 19(3):411-412. pmid: 10344330

14. Han S, Yoon H, Shin D, Song D. Trigger Thumb in Children: Results of Surgical Treatment in Children: Above 5 Years of Age. Journal of Pediatric Orthopaedics. 2010; 30(7):710-714. doi: https://doi.org/10.1097/BPO.0b013e3181edef8d

15. Watanabe $H$, Hamada $Y$, Toshima $T$, Nagasawa $K$. Conservative treatment for trigger thumb in children. Archives of Orthopaedic and Trauma Surgery. 2001; 121(7):388-390.

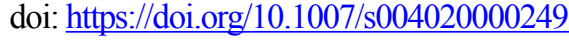

16. De Luna V, Potenza V, Garro L, Farsetti P, Caterini R. Multiple Congenital Bilateral Trigger Digits in a 2-Year-Old Child: Case Report. The Open Orthopaedics Journal. 2013; 7: 75-77. doi: https://doi.org/10.2174/1874325001307010075

17. Baek G, Lee Y. The Natural History of Pediatric Trigger Thumb: A Study with a Minimum of Five Years Follow-up. Clinics Orthopedic Surgery. 2011; 3(2):157-159. doi: https:// doi.org/10.4055/cios.2011.3.2.157

18. Forlin E, Kaetsu E, De Vasconcelos J. Success of conservative treatment of trigger thumb in children after minimum follow-up of five years. Revista Brasileira de Ortopedia. 2012; 47(4):4837. doi: https://doi.org/10.1016/S2255-4971(15)30133-6

19. Tan AH, Lam KS, Lee EH. The treatment outcome of trigger thumb in children. Journal of Pediatric Orthopaedics. 2002; 11(3):256-259. doi: https://doi.org/10.1097/00009957200207000-00011

20. Bae D. Pediatric Trigger Thumb. J Hand Surg Am. 2008; 33(7):1189-1191. doi: https://doi.org/10.1016/j. jhsa.2008.04.017

21. Ferreyra A, Schumacher F, Allende V, Masquijo J. Tratamiento quirúrgico del pulgar en resorte pediátrico. Revista Mexicana de Ortopedia Pediátrica. [Internet]. 2013[citado 14 de enero de 2019];2(15):105-110. Recuperado a partir de: https://www. medigraphic.com/pdfs/opediatria/op-2013/op132h.pdf

22. Van Loveren M, Van der Biezen J. The Congenital Trigger Thumb Is Release of the First Annular Pulley Alone Sufficient to Resolve the Triggering? Annals of Plastic Surgery. 2007; 58(3):335-337. doi: https://doi.org/10.1097/01. $\underline{\text { sap.0000238336.30617.72 }}$

23. Sevencan A, Inan U, Köse N, Omeroğlu H, Seber S. Percutaneous Release for Trigger Thumbs in Children: Improvements of the Technique and Results of 31 Thumbs. Journal of Pediatric Orthopaedics. 2010; 30(7):705-709. doi: https://doi.org/10.1097/BPO.0b013e3181efb90e 